\title{
Learning through the taste system
}

\author{
Thomas R. Scott* \\ Graduate and Research Affairs, San Diego State University, San Diego, CA, USA
}

Edited by:

Milagros Gallo, University of

Granada, Spain

Reviewed by:

Milagros Gallo, University of Granada, Spain

Edmund Rolls, University of

Oxford, UK

*Correspondence:

Thomas R. Scott, Graduate and

Research Affairs, San Diego State

University, 5500 Campanile Drive,

San Diego, CA, 92182-8220, USA.

e-mail: tom.scott@sdsu.edu
Taste is the final arbiter of which chemicals from the environment will be admitted to the body. The action of swallowing a substance leads to a physiological consequence of which the taste system should be informed. Accordingly, taste neurons in the central nervous system are closely allied with those that receive input from the viscera so as to monitor the impact of a recently ingested substance. There is behavioral, anatomical, electrophysiological, gene expression, and neurochemical evidence that the consequences of ingestion influence subsequent food selection through development of either a conditioned taste aversion (CTA) (if illness ensues) or a conditioned taste preference (CTP) (if nutrition). This ongoing communication between taste and the viscera permits the animal to tailor its taste system to its individual needs over a lifetime.

Keywords: taste, learning, conditioned taste aversion, conditioned taste preference, rat

\section{INTRODUCTION}

Taste is an intermediary between the external and internal worlds. It is located at the interface of these two vastly different environments, and thus is charged with making the final decision about what, from an uncontrolled and often hostile chemical surround, should be incorporated into the highly controlled biochemical environment within.

Taste is the beginning of a long chemosensory tube that extends from palate to intestines, with receptors along that length that are sensitive to the products liberated by digestion. Those on the palate are not unique to taste; the same receptors often occur elsewhere in the body. What is unique is that those serving taste gather their information before the irrevocable decision to swallow has been made, and so can influence that decision. Hence, whereas the distribution of information from other chemical sensors may be limited to the gastrointestinal (GI) tract, or may be conveyed through the vagus only to the hindbrain, that from taste receptors is projected through the brainstem to the thalamus and multiple cortical sites as well as to ventral forebrain areas. This vast distribution through the central nervous system permits the control of somatic and autonomic reflexes, a cognitive evaluation, and hedonic appreciation.

An apt metaphor for taste is a Janus head, mounted on an ancient city gate, one face turned outward to assess the traffic beyond the walls, warning of approaching toxins and alerting gatekeepers to the availability of nutrients; the other turned inward to monitor the city's changing needs and to adjust its decisions of what passes through the city walls to satisfy them (Scott, 1987).

This view of the role of gustation as a visceral (internal) as well as somatic (external) sense defines its learning capacity. Taste is exquisitely well suited to learn from visceral consequences (satiety, nausea); it is less inclined to learn from those that are somatic (tones, lights, and shocks). As Garcia noted in describing the development of a taste aversion following a meal, no other aspect of the event was implicated in having caused nausea: not his dinner companions, the table settings, or the background music, only the visceral component represented by the taste of the meal (Garcia et al., 1985).

Taste learning, then, is largely a matter of conditioning. The realm of conditioning can be broadly divided into those events that one can do something about, and those that one cannot. The former typically demands operant conditioning to manipulate the environment to one's satisfaction, using somatic senses to gather information and striated muscles for action; the latter more commonly demands classical conditioning to prepare for the inevitable, often using smooth muscles. Gustatory learning serves as a special case of classical conditioning, with taste representing the conditioned stimulus (CS), and the visceral sequelae of ingestion, the unconditioned stimulus (US). It has been proposed that the visceral response alters the reward value of the taste, and that this new value then guides the animal's behavior to either seek or avoid that taste (Rolls, 2005).

Such learning can occur in an appetitive or aversive direction, with the establishment of either conditioned taste aversions (CTAs) or preferences (CTPs).

Aversions are more robust. It is of greater urgency to the animal to avoid a chemical that has sickened it than to develop a preference for one among many that have proven to provide nutrition. The CTA is readily established with a single pairing of the gustatory CS and the visceral US, even with a CS-US interval for several hours. It is not impaired by placing the animal under deep anesthesia or rendering it comatose before the US is delivered. Indeed, the predisposition of an animal to develop a CTA is so striking that the investigators first suspected it to be an artifact, a suspicion laid to rest only by an exhaustive series of studies and arguments (Revusky, 1977).

As easily as it is created, a CTA is notoriously difficult to extinguish (Nolan et al., 1997). Having been poisoned is clearly an experience not to be forgotten. The CTA also has a robust impact on behavior, often suppressing subsequent acceptance of the CS to less than $10 \%$ of preconditioned levels, even in animals motivated to consume by moderate deprivation. With such a 
dramatic impact on behavior, the CTA has a half-century history as a rich topic of research. Thousands of studies were conducted during the 1960s and 1970s on behavioral variables such as the distinctiveness and novelty of the taste, the nature and severity of the nausea, the amount of time between them and how that time was spent. With these clearly defined, the CTA could be employed by researchers as a tool for altering taste acceptability, creating a profound reduction in acceptance of the CS, from which generalization gradients of both quality and intensity could be determined to reveal the relative similarities among taste qualities.

In parallel, behavioral neuroscientists began to investigate the mechanisms by which this extraordinary learning process occurred, using rats in nearly all studies. They performed lesions of taste pathways and relays to determine which areas of the nervous system were required in order to develop and retain a CTA. There followed electrophysiological investigations of the impact of a CTA on taste processing, immunohistochemical studies of gene expression elicited by a CTA, and microdialysis experiments on the neurochemical consequences of the experience.

The modest counterpart of the CTA - the CTP-has received less attention. A CTP can be established rather quickly by pairing a novel taste with recovery from a dietary deficiency, most notably the provision of thiamine to animals on a thiamine-deficient diet (Rodgers, 1967; Capretta, 1977). More commonly, however, the impact of a CTP on behavior is revealed only gradually over days of continuous pairing of taste with nutrition, though that impact can reach levels equal to those of a CTA in the opposite direction, i.e., approaching 100\% preference (Sclafani and Nissenbaum, 1988). The electrophysiological and neurochemical concomitants of a CTP are also more subtle than those of a CTA. Yet, the CTP may have played a larger role in defining human culture than the CTA, for while the latter is powerful, it is idiosyncratic to the individual. The CTP, by contrast, is often shared by members of a culture where certain foods are available. It is typical of a culture's cuisine that there are a few piquant tastes (the CS) accompanied by carbohydrate loads (the US). The gustatory CS comes to be favored by association with the nutritional US, and the cuisine, with all its cultural trappings and traditions, tends to bind its consumers together as part of their cultural identity.

In the paragraphs that follow, I will review some of the work on the mechanisms of CTAs and CTPs that have come from our laboratory and those of our colleagues.

\section{CONDITIONED TASTE AVERSION: LESION STUDIES}

The ingredients of the CTA - taste and visceral distress-are represented widely across the CNS. Investigators have performed lesions of areas that receive such inputs in an effort to define which are crucial to the creation and to subsequent retention of a CTA. Results implicate the area postrema in acquisition (Rabin et al., 1983a), but not retention (Rabin et al., 1983b). They reveal that loss of the parabrachial nuclei (PBN) causes the most profound disruption on both creating a CTA (with lesions of the lateral division) (Spector et al., 1992; Yamamoto et al., 1994) and maintaining a previous aversion (medial division) (Sakai et al., 1994). They implicate the ventromedial globus pallidus in both acquisition and retention (Hernadi et al., 1997). Electrolytic lesions of the basolateral amygdala disrupt the creation and retention of a CTA (Yamamoto et al., 1995), but NMDA lesions, which spare fibers of passage, do not (Chambers, 1990); thus the amygdala remains a likely participant in CTA formation, but the axons that pass through it may be of greater import, since leaving them intact sustains the learning. Lesions of prefrontal cortex have yielded conflicting results, and its role in CTAs remains uncertain. Those in insular cortex (IC), however, reliably degrade CTA acquisition (Braun et al., 1982; Bermudez-Rattoni and McGaugh, 1991) and have an even larger impact on retention. Thus, the cast of participants in creating and retaining a CTA as demonstrated by these fixed, permanent lesions range from the deepest recesses of the brain stem through ventral forebrain to the neocortex.

Greater insight may be gleaned from lesions that are reversible, or that combine the loss of more than one area. Ivanova and Bures (1990a,b) temporarily disabled regions of the brainstem with microinjections of tetrodotoxin (TTX). They reported that TTX injected in the PBN up to one day in advance or four days following training blocked the consolidation of a CTA without affecting the rejection threshold for quinine. Thus, taste processing per se remained intact, but the associative functions required for learning the aversion were disrupted by inactivation of the PBN.

The crucial role of $\mathrm{PBN}$ in mediating associative taste learning was reinforced by Clark and Bernstein (2009). These investigators performed asymmetrical, unilateral lesions of the PBN and IC, and thereby disrupted CTA acquisition. However, when both lesions were made in the same hemisphere, leaving the contralateral hemisphere intact, learning proceeded normally. Thus, communication between PBN and IC is essential for CTA learning.

\section{CONDITIONED TASTE AVERSION: ELECTROPHYSIOLOGY}

The effect of a CTA is to reverse the behavioral reaction to a previously preferred taste to one of the revulsion. The rejection response is organized in caudal brainstem (Norgren and Grill, 1982) and released in stereotypical fashion upon encountering an inherently aversive stimulus or one to which a CTA has been formed. The nucleus of the solitary tract (NST), the first central relay for taste, is likely to be involved in CTA formation. Here gustatory and visceral afferents converge (Norgren, 1981) yet do not directly overlap, communicating instead via the adjacent reticular formation, offering a close association between signals from the two necessary components of a CTA.

Chang and Scott (1984) took single neuron recordings from the NST of rats that were (1) unconditioned (tasted the saccharin CS with no subsequent nausea), (2) pseudoconditioned (experienced the nausea US with no preceding taste), or (3) conditioned (the taste of the saccharin CS was paired with $\mathrm{LiCl}-$ induced nausea US). The recordings revealed that sweet-oriented NST neurons gave exaggerated responses to the saccharin CS in conditioned rats, and that the increase was due to a sharp spike of activity that peaked at about $900 \mathrm{msec}$ following stimulus delivery (Figure 1). This is reminiscent of the phasic burst of activity elicited by aversive quinine. Moreover, the neural response profile to saccharin in conditioned rats was more similar to those of 


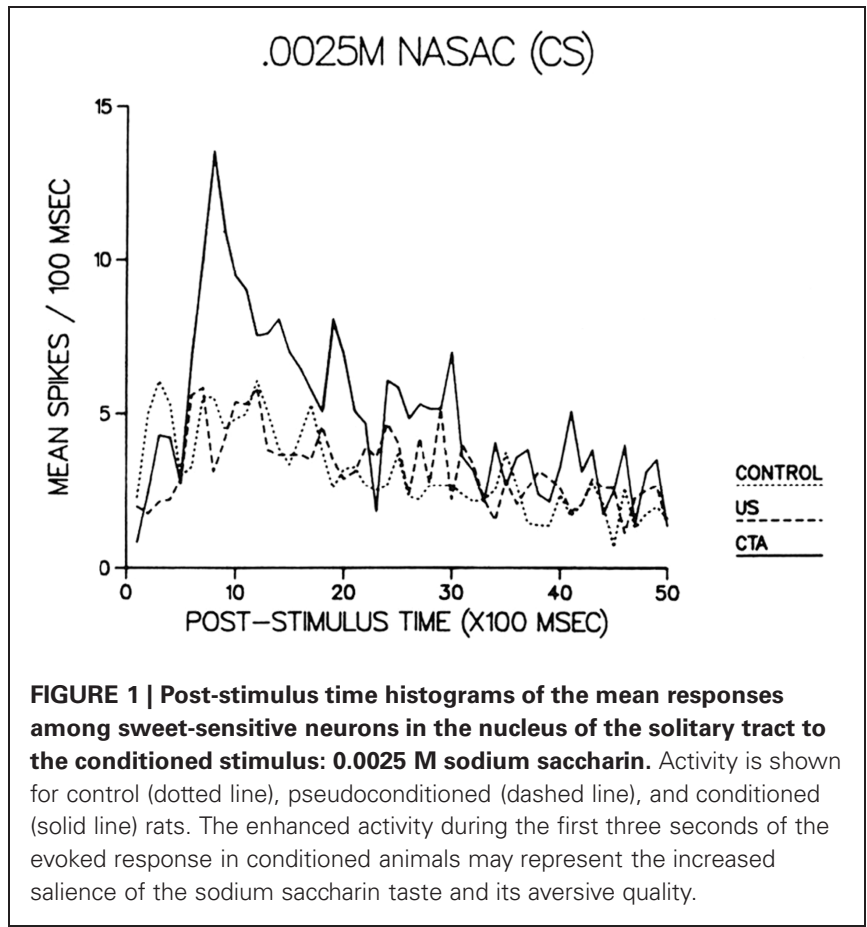

aversive stimuli. We concluded that the sensory code for the saccharin CS (and, to a lesser extent, for other sweet stimuli) was altered at the first central taste relay by conditioning, and that such a modification could explain not only the behavioral reaction, but also the immunohistochemical, and neurochemical consequences of a CTA described below. Such a modification of the taste signal might also reveal why the cephalic phase insulin release from the pancreas, a parasympathetic reflex elicited by sweet taste, is blocked after a CTA has been created to that taste (Louis-Sylvestre and LeMagnen, 1980). The altered neural message would lose the capacity to innervate the vagal efferents responsible for stimulating pancreatic $\beta$ cells.

In a subsequent experiment, we created, and then fully extinguished a CTA to saccharin (Nolan et al., 1997), then recorded responses to saccharin and other stimuli in these recovered rats and in unconditioned controls (McCaughey et al., 1997). The mean responses to all stimuli were no different in the two groups of rats, nor was there any significant difference between the neural response profiles to any taste. However, the neural activity was not completely restored to the preconditioning state. There remained a clear vestige of the conditioning experience in an attenuated burst given to the CS by the sweet-sensitive subgroup of neurons. The burst was no longer associated with conditioned behaviorwhich was fully extinguished-though it may have served as a permanent marker for a once-salient CS that can abet subsequent reacquisition of the aversion.

Curiously, lesions in gustatory NST do not interfere with either the acquisition or retention of a CTA (Grigson et al., 1997; Shimura et al., 1997a). Even if the electrophysiological effects recounted above were only advisory to a more commanding CTA nucleus or circuit, the blockade of taste information at this obligatory synapse would appear to prevent any subsequent learning. The lack of an impact may reflect the inadequacy of lesions to fully compromise a functioning relay, particularly when they must spare adjacent areas that control vital reflexes, including respiration.

In the parabrachial nucleus, the creation of a CTA to $\mathrm{NaCl}$ resulted in an elevated response to that stimulus in sodiumspecific taste cells, in agreement with the responses to the saccharin CS in the NST described above (Shimura et al., 1997b). The same was found in the IC (Yamamoto et al., 1989) and amygdala, where an exaggerated response to the CS is often expressed as inhibition (Yasoshima et al., 1995). Finally, in the hypothalamus of naive rats, the taste of saccharin activates areas associated with feeding and inhibits those for satiety; after the saccharin is paired with nausea, these roles are reversed (Aleksanyan et al., 1976). Thus, the impact of a CTA is demonstrable in the electrophysiological activity of neurons across the widely dispersed regions that process taste activity, and that impact is appropriate to guide the aversive reaction to the CS that follows conditioning.

\section{CONDITIONED TASTE AVERSION: GENE EXPRESSION}

The formation of long-term memories requires the expression of immediate early genes and the synthesis of their associated proteins (McGaugh, 2000). The gene $c$-fos has been shown to be expressed and the associated Fos protein synthesized in a variety of species as a basis for modifying the neural activity associated with learning (Sanyal et al., 2002). c-fos expression, then, can serve as a useful index of conditioning.

Houpt et al. (1994) demonstrated that sucrose elicited $c$-fos expression in the NST after it had been paired with an intraperitoneal injection of $\mathrm{LiCl}$. This was not simply a response to the aversive taste that sucrose had become, for quinine did not induce the same expression in unconditioned rats (Houpt et al., 1996). However, this index of learning in NST was blocked when the amygdala was impaired, demonstrating the importance of centrifugal fibers in mediating the conditioning process (Schafe and Bernstein, 1996).

The results of gene expression are more detailed in the PBN. Yamamoto and his colleagues have shown that $c$-fos is induced in cells in the medial division of PBN by hedonically positive tastes associated with ingestion, and in neurons in more lateral divisions by aversive tastes. Saccharin activates medial cells in naïve rats, but after the saccharin has been paired with a $\mathrm{LiCl}$ injection, its taste induced $c$-fos expression in the lateral division (Yamamoto et al., 1994). Moreover, when $c$-fos expression was blocked in PBN, CTA learning was impaired, just as it is when the PBN is lesioned (Yasoshima et al., 2006). Thus, a functioning PBN, capable of modifying its responses to a taste stimulus as a consequence of experience, is crucial to learning to avoid toxins.

In forebrain, Bernstein and Koh (2007) addressed the issue of which areas reacted to novel tastes differently from those that were familiar, since only the former serve as effective conditioned stimuli. They used $c$-fos expression to identify the central nucleus of the amygdala (CNA) and IC as two such sites. The next question was which of these also responded to the US, the second of the necessary elements of a CTA. Only the CNA expressed $c$-fos to a $\mathrm{LiCl}$ US, implicating this nucleus as 
a crucial nexus for making the association. Yet while IC, which had responded to gustatory novelty, did not show increased $c$-fos expression to the US alone, its neurons did respond to pairing of the CS and US, reinforcing its role in the associative process, perhaps through communication with the PBN and amygdala (Ferreira et al., 2006).

Gene expression, of course, is a precursor of protein production, and it is assumed that the protein is the basis for forming the associative memory. Accordingly, when protein synthesis was inhibited by administration of anisomycin into the IC, a CTA was not formed (Rosenblum et al., 1993). More specifically, the administration of oligodeoxynucleotide (ODN) antisense to $c$-fos in the amygdala blocked CTA acquisition (Lamprecht and Dudai, 1996). Again, the two critical forebrain regions-IC and amygdala-are implicated, and the capacity of their neurons to synthesize proteins in general, and Fos in particular, is essential to associative learning.

Finally, Bernstein and her colleagues (Barot et al., 2008; Chung et al., 2011) used a direct visualization approach to identify neurons that responded both to the CS and US, reasoning that this afforded them prima facie data from which to support the association. They found such cells only in the basolateral nucleus of the amygdala, and only under normal training conditions, i.e., CS followed by US.

The bulk of evidence from gene expression studies, then, focuses on three structures as being central to the acquisition and retention of CTAs: the PBN, the amygdala (both central and basolateral nuclei), and the IC.

\section{CONDITIONED TASTE AVERSION: NEUROCHEMISTRY}

Experiencing the conditions under which a CTA is created is stressful. It is unsurprising, then, that when the CS to which a CTA has been developed is subsequently presented, reminiscent of that experience, plasma corticosteroid levels rise (Smotherman et al., 1976). Yet, the CTA does not depend on this adrenal index of stress, for adrenalectomized rats acquired CTAs as readily as controls (Ader et al., 1978).

A more specific measure of the impact of a CTA is seen in the levels of neurochemicals associated with reward or aversion, particularly in the limbic system. Reward is associated with increased dopamine (Hoebel, 1984) and reduced acetylcholine (Rada et al., 1991) in the nucleus accumbens, and lowered serotonin levels in the paraventricular hypothalamus (Stanley et al., 1989). This relationship has been demonstrated both through microdialysis, where dopamine levels in the accumbens rose following a sweet taste, and conversely through reverse microdialysis in which dopamine administered to the accumbens increased sucrose consumption (Hajnal and Norgren, 2001).

\section{REFERENCES}

Ader, R., Grota, L. J., and Buckland, R. (1978). Effects of adrenalectomy on taste aversion learning. Physiol. Psychol. 6, 359-361.

Aleksanyan, A., Buresova, O., and Bures, J. (1976). Modification of unit responses to gustatory stimuli by conditioned taste aversions in rats. Physiol. Behav. 17, 173-179.

Barot, S. K., Kyomo, Y., Clark, E. W., and Bernstein, I. L. (2008). Visualizing stimulus convergence in amygdala neurons during associative learning. PNAS, 105, 20,959-20,963.

The taste of saccharin evoked dopamine release in the rat's accumbens, in accord with its reinforcing value. However, if the saccharin had been paired with nausea to create a CTA, the same stimulus caused a reduction in dopamine, and instead a release of acetylcholine in accumbens (Mark et al., 1991), and serotonin in the hypothalamus (West et al., 1991), denying the neurochemical basis for reward.

\section{CONDITIONED TASTE PREFERENCE}

When subjects develop a CTA, there is little doubt that they are subsequently repulsed by the taste. Not only do they avoid it, they also show the well-defined mimetic reflexes of aversiveness: gaping, head-shaking, and chin-rubbing. All that follows-the blockade of parasympathetic reflexes, the alteration of the afferent signal and its projection to brain areas associated with avoidance, the reversal of neurochemical release from rewarding to aversive-is in accord with this powerful experience.

The impact of a CTP is more subtle. The behavior changes only over days of training, though the CS does finally reach asymptotically high levels of acceptance, and is quite resistant to extinction. Mimetic responses of rats, however, do not change as the CS wins acceptance, raising the question of whether the hedonic quality of the CS has increased (Sclafani, 1991). Two types of evidence argue that it does. Giza et al. (1997) recorded electrophysiological responses from the NST of rats that had been conditioned to prefer either of two formerly aversive chemicals. In each case, the gustatory code was modified to reflect a less aversive quality, though the effect was less pronounced than that seen in the opposite direction upon creation of a CTA (Chang and Scott, 1984). Secondly, the taste for which a preference was acquired now elicited a heightened dopamine release in the nucleus accumbens, a clear neurochemical marker of reward (Mark et al., 1994).

\section{CONCLUSION}

Learning through the taste system is intimately allied with GI consequences. The animal knows two facts: what the chemical was (taste), and what it did (GI). This information permits it to tailor its chemical selection to full individual advantage over a lifetime. The learning process draws on responses that extend from the viscera through caudal brainstem, to ventral forebrain and cortex, implying an ancient system, much like the control of feeding itself. It is only a marginally conscious process, for CTAs can be learned while comatose, and most people cannot recall the occasion upon which they developed a food aversion (Bernstein, 1985). Conditioned aversions and preferences provide the operative link between the chemical and biochemical environments.

Bermudez-Rattoni, F., and McGaugh, J. L. (1991). Insular cortex and amygdala lesions differentially affect acquisition on inhibitory avoidance and conditioned taste aversion. Brain Res. 549, 165-170.

Bernstein, I. L. (1985). Learned food aversions in the progression of cancer and its treatment. Ann. N. Y. Acad. Sci. 443, 365-380.

Bernstein, I. L., and Koh, M. T. (2007). Molecular signaling during taste aversion learning. Chem. Senses 32, 99-103.

Braun, J. J., Lasiter, P. S., and Kiefer, S. W. (1982). The gustatory neocortex 
of the rat. Physiol. Psychol. 10, 13-45.

Capretta, P. J. (1977). "Establishment of food preferences by exposure to ingestive stimuli early in life," in Learning Mechanisms in Food Selection, eds L. M. Barker, M. R. Best, and M. Domjan (Waco, TX: Baylor University Press), 99-121.

Chambers, K. C. (1990). A neural model for conditioned taste aversions. Annu. Rev. Neurosci. 13, 373-385.

Chang, F-C. T., and Scott, T. R. (1984). Conditioned taste aversions modify neural responses in the rat nucleus tractus solitarius. J. Neurosci. 4, 1850-1862.

Chung, A., Barot, S. K., Kim, J. J., and Bernstein, I. L. (2011). Biologically predisposed learning and selective associations in amygdalar neurons. Learn. Mem. 18, 391-394.

Clark, E. W., and Bernstein, I. L. (2009). Establishing aversive, but not safe, taste memories requires lateralized pontine-cortical connections. Behav. Brain Res. 197, 356-363.

Ferreira, G., Ferry, B., Meurisse, M., and Lúvy, F. (2006). Forebrain structures specifically activated by conditioned taste aversion. Behav. Neurosci. 120, 952-962.

Garcia, J., Lasiter, P. S., BermúdezRattoni, F., and Deems, D. A. (1985). "A general theory of aversion learning," in Experimental Assessments and Clinical Applications of Conditioned Food Aversions, eds M. S. Braveman and P. Bronstein, (Ann. N.Y. Acad. Sci.) (New York, NY), 443, 8-21.

Giza, B. K., Ackroff, K., McCaughey, S. A., Sclafani, A., and Scott, T. R. (1997). Preference conditioning alters taste responses in the nucleus of the solitary tract of the rat. Am. J. Physiol. 273, R1230-R1240.

Grigson, P. S., Shimura, T., and Norgren, R. (1997). Brainstem lesions and gustatory function: III. The role of the nucleus of the solitary tract and the parabrachial nucleus in retention of a conditioned taste aversion in rats. Behav. Neurosci. 111, 180-187.

Hajnal, A., and Norgren, R. (2001). Accumbens dopamine mechanisms in sucrose intake. Brain Res. 904, 76-84.

Hernadi, I., Karadi, Z., Faludi, B., and Lenard, L. (1997). Disturbances of neophobia and taste aversion learning after bilateral kainite microlesions in the rat pallidum. Behav. Neurosci. 111, 137-146.

Hoebel, B. G. (1984). "Neurotransmitters in the control of feed- ing and its rewards: monoamines, opiates, and brain-gut peptides," in Eating and Its Disorders, eds A. J. Stunkard and E. Stellar (New York: Raven Press), 15-38.

Houpt, T. A., Philopena, J. M., Wessel, T. C., Joh, T. H., and Smith, G. P. (1994). Increased $c$-fos expression in nucleus of the solitary tract correlated with conditioned taste aversion to sucrose in rats. Neurosci. Lett. 172, 1-5.

Houpt, T. A., Philopena, J. M., Wessel, T. C., Joh, T. H., and Smith, G. P. (1996). c-fos induction in the rat nucleus of the solitary tract by intraoral quinine infusion depends on prior contingent pairing of quinine and lithium chloride. Physiol. Behav. 60, 1535-1541.

Ivanova, S. F., and Bures, J. (1990a). Acquisition of conditioned taste aversion in rats is prevented by tetrodotoxin blockade of a small midbrain region centered around the parabrachial nuclei. Physiol. Behav. 48, 543-549.

Ivanova, S. F., and Bures, J. (1990b) Conditioned taste aversion is disrupted by prolonged retrograde effects of intracerebral injection of tetrodotoxin in rats. Behav. Neurosci. 104, 948-954.

Lamprecht, R., and Dudai, Y. (1996). Transient expression of $c$-fos in rat amygdala during training is required for encoding conditioned taste aversion memory. Learn. Mem. 3, 31-41.

Louis-Sylvestre, J., and LeMagnen, J. (1980). Palatability and preabsorptive insulin release. Neurosci. Biobehav. Rev. 4, 43-46.

Mark, G. P., Blander, D. S., and Hoebel, B. G. (1991). A conditioned stimulus decreases extracellular dopamine in the nucleus accumbens after development of a learned taste aversion. Brain Res. 551, 308-310.

Mark, G. P., Smith, S. E., Rada, P. V., and Hoebel, B. G. (1994). An appetitively conditioned taste elicits a preferential increase in mesolimbic dopamine release. Pharmacol. Biochem. Behav. 48, 651-660.

McCaughey, S. A., Giza, B. K., Nolan, L. J., and Scott, T. R. (1997). Extinction of a conditioned taste aversion in rats: II. Neural effects in the nucleus of the solitary tract. Physiol. Behav. 61, 373-379.

McGaugh, J. (2000). Memory-A century of consolidation. Science, 287, 248-251.

Nolan, L. J., McCaughey, S. A., Giza, B. K., Rhinehart-Doty, J. A., Smith, J. C., and Scott, T. R. (1997). Extinction of a conditioned taste aversion in rats: I. Behavioral effects. Physiol. Behav. 61, 319-323.

Norgren, R. (1981). "The central organization of the gustatory and visceral afferent systems in the nucleus of the solitary tract," in Brain Mechanisms of Sensation, eds Y. Katsuki, R. Norgren, and M. Sato, (New York: John Wiley and Sons) 143-160.

Norgren, R., and Grill, H. J. (1982). "Brainstem control of ingestive behavior," in Physiological Mechanisms of Sensation, ed. D. W. Pfaff (Berlin: Springer Verlag), 99-131.

Rabin, B. M., Hunt, W. A., and Lee, J. (1983a). Attenuation of radiation and drug-induced conditioned taste aversions following area postrema lesions in the rat. Radiat. Res. 93, 388-394.

Rabin, B. M., Hunt, W. A., and Lee, J. (1983b). Recall of a previously acquired conditioned taste aversion in rats following lesions of the area postrema. Physiol. Behav. 32, 119-122.

Rada, P., Mark, G. P., Pothos, E., and Hoebel, G. G. (1991). Systemic morphine simultaneously decreases extracellular acetylcholine and increases dopamine in the nucleus accumbens of freely moving rats. Neuropharmacology 30, 1133-1136.

Revusky, S. (1977). "Interference with progress by the scientific establishment: examples from taste aversion learning," in Food Aversion Learning, eds N. Milgram, L. Krames, and T. Alloway (New York: Plenum Press), 53-71.

Rodgers, W. L. (1967). Specificity of specific hungers. J. Comp. Physiol. Psychol. 64, 49-58.

Rolls, E. T. (2005). Emotions Explained. Oxford, UK: Oxford University Press.

Rosenblum, K., Meiri, N., and Dudai, Y. (1993). Taste memory: the role of protein synthesis in gustatory cortex. Behav. Neural Biol. 59, 49-56.

Sakai, N., Tanimizu, T., Sako, N. Shimura, T., and Yamamoto, T. (1994). "Effects of lesions of the medial and lateral parabrachial nuclei on acquisition and retention of conditioned taste aversion,' in Olfaction and Taste XI, eds K. Kurihara, N. Suzuki, and H. Ogawa (Tokyo: Springer Verlag), 495-496.

Sanyal, S., Sandstrom, D. J., Hoeffer, C. A., and Ramaswami, M. (2002). AP-1 functions upstream of CREB to control synaptic plasticity in drosophila. Nature, 416, 870-874.
Schafe, G. E., and Bernstein, I. L. (1996). Forebrain contribution to the induction of a brainstem correlate of conditioned taste aversion: I. The amygdala. Brain Res. 741, 109-116.

Sclafani, A. (1991). Conditioned food preferences. Bull. Psychonom. Soc. 29, 256-260.

Sclafani, A., and Nissenbaum, J. W. (1988). Robust conditioned flavor preference produced by intragastric starch infusions in rats. Am. J. Physiol. 255, R672-R675.

Scott, T. R. (1987). "The Janus head of taste," in Olfaction and Taste IX, eds S. D. Roper and J. Atema (New York: The New York Academy of Sciences), 600-601.

Shimura, T., Norgren, R., and Grigson, P. S. (1997a). Brain lesions and gustatory function: I. The role of the nucleus of the solitary tract during a brief intake test in rats. Behav. Neurosci. 111, 155-168.

Shimura, T., Tanaka, H., and Yamamoto, T. (1997b). Salient responsiveness of the parabrachial neurons to the conditioned stimulus after the acquisition of taste aversion learning in rats. Neuroscience 81, 239-247.

Smotherman, W. P., Hennessey, J. W., and Levine, S. (1976). Plasma corticosterone levels during recovery from $\mathrm{LiCl}$ produced taste aversions. Behav. Biol. 16, 401-412.

Spector, A. C., Norgren, R., and Grill, H. J. (1992). Parabrachial gustatory lesions impair taste aversion learning in rats. Behav. Neurosci. 106, 147-161.

Stanley, B. G., Schwatz, D. H., Hernandez, L., Liebowitz, S. F., and Hoebel, B. G. (1989). Patterns of extracellular 5-hydroxyindoleacetic acid (5-HIAA) in the paraventricular hypothalamus: relation to circadian rhythm and deprivationinduced eating behavior. Pharmacol. Biochem. Behav. 33, 257-260.

West, H. L., Mark, G. P., and Hoebel, B. G. (1991). Effect of conditioned taste aversion on extracellular serotonin in the lateral hypothalamus and hippocampus of freely moving rats. Brain Res. 556, 95-100.

Yamamoto, T., Matsuo, R., Kiyomitsu, Y., and Kitamura, R. (1989). Taste responses of cortical neurons in freely ingesting rats. J. Neurophysiol. 61, 1244-1258.

Yamamoto, T., Fujimoto, Y., Shimura, T., and Sakai, N. (1995). Conditioned taste aversion in rats with excitotoxic brain lesions. Neurosci. Res. 22, 31-49.

Yamamoto, T., Shimura, T., Sakai, N., and Ozaki, N. (1994) Representation of hedonics and 
quality of taste stimuli in the parabrachial nucleus of the rat. Physiol. Behav. 56, 1197-1202.

Yasoshima, Y., Sako, N., Senba, E. and Yamamoto, T. (2006). Acute suppression, but not chronic genetic deficiency, of $c$-fos gene expression impairs long-term memory in aversive taste learning. PNAS 103, 7106-7111.
Yasoshima, Y., Shimura, T., and Yamamoto, T. (1995). Single unit responses of the amygdala after conditioned taste aversion in conscious rats. Neuroreport, 6, 2424-2428.

Conflict of Interest Statement: The authors declare that the research was conducted in the absence of any commercial or financial relationships that could be construed as a potential conflict of interest.

Received: 28 July 2011; accepted: 10 October 2011; published online: 23 November 2011.

Citation: Scott TR (2011) Learning through the taste system. Front. Syst.
Neurosci. 5:87. doi: 10.3389/fnsys 2011.00087

Copyright (c) 2011 Scott. This is an open-access article subject to a nonexclusive license between the authors and Frontiers Media SA, which permits use, distribution and reproduction in other forums, provided the original authors and source are credited and other Frontiers conditions are complied with. 